\title{
INFORMATION CONSERVATION, ENTROPY INCREASE AND THE STATISTICAL IRREVERSIBILITY FOR AN ISOLATED SYSTEM
}

\author{
Qi-Ren Zhang \\ Department of Technical Physics, Peking University, Beijing,100871, China
}

November 13, 2018

\begin{abstract}
We consider the statistical irreversibility and its compatibility with the reversible dynamics. The role played by the observation is analyzed in detail. It makes our previous proof for the second law of thermodynamics clearer. On this basis, we emphasize the importance and wide applicability for the second law of thermodynamics. A new form of physics with this law being substituted by the principle of information conservation is suggested. By the way, we also solve the paradox of Schrödinger cat, and show that the universe will not go to the so-called heat death spontaneously.
\end{abstract}

PACS: 05.30.Ch, 05.70.-a, 03.65.-w

Keywords: Information conservation, Information change in observation, Entropy increase, Statistical irreversibility

\section{Introduction}

We proved the second law of thermodynamics exactly $[1,2]$, from the principle of information conservation, which is exactly valid both in classical and quantum dynamics. However the proof was purely mathematical. Here we would complete it with a physical analysis to make the proof qualitatively understandable. Then we may say that the problem of understanding this law on the basis of dynamics and statistics is completely solved. The analysis shows, if the dynamics is reversible, the irreversibility of a macroscopic process may arise because of the change of information and the corresponding change of statistic ensemble in the observation. We call this kind of irreversibility statistical. 


\section{Information and its change in observations}

For any probability distribution $\left[W_{n}\right]$ one may define its information

$$
\mathcal{I}=\sum_{n} W_{n} \ln W_{n}
$$

After an observation, the probability distribution and therefore the information changes. For an example, in a complete measurement one finds that the possibility $n=n_{0}$ is realized, and all other possibilities of $n \neq n_{0}$ disappear. The probability distribution becomes $W_{n_{0}}=1$, $W_{n \neq n_{0}}=0$, and the information becomes $\mathcal{I}_{0}=0$. The observer gains the information $\mathcal{I}_{0}-\mathcal{I}=$ $-\mathcal{I}=-\sum_{n} W_{n} \ln W_{n} \geq 0$. There are also observations, in which the observer loses information.

Consider a set $[|n\rangle]$ of independent states for a system, in which $|n\rangle$ is the $n$-th state in the set. The set, with a probability distribution $\left[W_{n}\right]$ defined on it, is called a statistic ensemble of the system. Substituting the distribution $\left[W_{n}\right]$ into (1), the obtained $\mathcal{I}$ is called the information of the system. Divide the system into subsystems. The probability distributions on the sets of independent states for subsystems are obtained by reducing the probability distribution of the system, and the information of subsystems is defined. There is an exact mathematical inequality $[1,3]$

$$
\sum_{i} \mathcal{I}_{i} \leq \mathcal{I}
$$

in which $\mathcal{I}_{i}$ is the information of the $i$-th subsystem, the summation is over all subsystems, and the equality holds if and only if the probability distribution for the system is factorized into a direct product of the probability distributions for subsystems. When one observes the system by measuring its subsystems separately, he ignores the correlation between the subsystems, the probability distribution for the system is therefore factorized. He gets the information $\mathcal{I}_{0}=\sum_{i} \mathcal{I}_{i}$ for the system. According to (2), the observer usually loses information in this kind of observation.

\section{Information conservation and the entropy increase}

The states of the system evolve in time according to the dynamics. For an isolated system, independent states always evolve continuously into independent states. This is true both in quantum and in classical dynamics. It means the probability distribution on independent states does not change in time, only the states on which the probability distribution is to be defined may be changed. Therefore the information of the isolated system does not change in time either. This is the information conservation. It is a fundamental principle both for quantum and classical physics.

Entropy is an extensive variable. The entropy of a system is therefore defined by the sum of entropies of its individual subsystems. The entropy of an individual subsystem is defined by its negative information in the unit of Boltzmann constant. The entropy of the system in this unit 
is therefore defined by[4]

$$
S \equiv-\sum_{i} \mathcal{I}_{i}
$$

in which $\mathcal{I}_{i}$ is the information of the $i$-th subsystem, and the summation is over all of its subsystems. From (2) we see that the entropy and the information for a system are related by $S \geq-\mathcal{I}$. If one wants to determine the entropy of the system at an initial time $t_{0}$, he has to measure the entropy of each individual subsystem. In this observation he loses the correlation information between the subsystems. The obtained entropy at this time is therefore $S\left(t_{0}\right)=-\sum_{i} \mathcal{I}_{i}\left(t_{0}\right)=-\mathcal{I}\left(t_{0}\right)$. From time $t_{0}$ to time $t$, the system evolves according to its dynamics with information conservation. It means that the information of the system at time $t$ is $\mathcal{I}(t)=\mathcal{I}\left(t_{0}\right)$, and the entropy of the system at time $t$ is therefore

$$
S(t) \geq-\mathcal{I}(t)=-\mathcal{I}\left(t_{0}\right)=S\left(t_{0}\right)
$$

It shows that the entropy of an isolated system strictly increases between two successive observations, unless the probability distribution for the system keeps being factorized at time t, it is that there is no correlation between these subsystems. In the later case the entropy does not change, and the system is in equilibrium. This is the principle of entropy increase. The factorization condition of probability distribution is just the start point for the derivation of Gibbs distribution for equilibrium systems[4].

\section{Ensemble change and the statistical irreversibility}

The observer gains or loses information in an observation, therefore he has to redefine the statistic ensemble after the observation, just like a general changes his estimation on the war situation by receiving a telegram from the front. In our case, the ensemble of the system is considered. The states in ensemble $\mathrm{A}$ at time $t_{0}$ evolve into the states in ensemble $\mathrm{B}$ at time $t$, according to the dynamics of the system. If the dynamics is reversible, the time inverse ensemble B' of B, obtained by changing every state in $\mathrm{B}$ to its time inverse state, evolves from time $-t$ to time $-t_{0}$ into the time inverse ensemble $\mathrm{A}^{\prime}$ of $\mathrm{A}$. However, an observation on the system at time $t$ changes the ensemble from $\mathrm{B}$ to $\mathrm{C}$, its time inverse ensemble $\mathrm{C}$ ' at $-t$ does not evolve into the ensemble $A^{\prime}$ at $-t_{0}$ in general. This is the statistical irreversibility. We see that it is perfectly compatible with the dynamical reversibility.

The consideration of observation in quantum physics needs more caution, because of the so-called wave packet collapse. It is assumed that a superposition of partial waves suddenly collapses into one of its partial waves when a measurement is performed, and the process is irreversible. Since there is not a clear partition between the measured system, the measuring instrument, and the observer, one may assume that these three parts consist a single macroscopic system. When one considers the wave packet collapse of this kind of system, he immediately falls into the discomfiture of the so called Schrödinger cat. The way to escape from this discomfiture 
is to recognize that the wave function is a probabilistic description of the system. Every partial wave corresponds to a set of possible properties, and the relative strengths of the partial waves denote the probability distribution on the sets of possible properties. When these partial waves superpose in a common configuration space, they interfere each other. But it becomes more and more difficult to make two objects superpose when the dimension of space increases. To understand it, let us consider a model, in which a point moves in a $f$-dimensional space of volume $L^{f}$, and ask the probability of the point passing through a small box of volume $l^{f}$ in this space, when it moves from one side of the space to another side. This model may be used to simulate the coincidence of two partial waves in a $f$-dimensional configuration space. If the motion is in random, the coincidence probability is $(l / L)^{f-1}$, which decreases exponentially with the dimension $f$; and the time one has to wait for seeing this coincidence is as long as $T \geq(L / l)^{f-1} L / c$, which increases exponentially with $f, c$ is the speed of light. Take $L=$ 1micron, it is not big, and $l / L=0.01$, it is not too small. We see, for $f=18$, which is the degrees of freedom for 6 particles in a 3 -dimensional space, the coincidence probability is $10^{-34}$, it is practically zero; and $T \geq 3 \times 10^{19}$ sec, it is longer than the universe age and means people have never seen this kind of coincidence since the birth of the universe, or it has never happened. Based on this understanding, one may define the measurement on a system to be an interaction between this system and a macroscopic system, usually (but not always) composed of the measuring instrument and the observer, which makes the wave packet of the total system decompose into a number of partial waves separated by macroscopic distance in their configuration space of numerous degrees of freedom. This is a purely objective process governed by the usual physical laws. Since these partial waves are practically impossible to meet each other once again, they cannot interfere each other any more. At the end, the observer finds that the properties corresponding to one of those partial waves is realized, and this result will appear repeatedly in his following observations on the same system. The assumption of a physical wave packet collapse is therefore unnecessary. As in the classical case, The observer has to change his information and the statistic ensemble for the system, from that described by the original wave packet to that described by the partial wave, which he read off from the measuring instrument. This change leads to the statistical irreversibility. By the way, the paradox of Schrödinger cat is solved. Before the observer sees it, the cat was either alive or dead after the corresponding partial waves being macroscopically separated and practically not able to interfere each other any more. Nothing is strange. This argument offers a concrete explanation on the role played by observation in above proof for the principle of entropy increase at the quantum level.

\section{$5 \quad$ Meanings and applications}

The sum $\mathcal{I}_{0} \equiv \sum_{i} \mathcal{I}_{i}$ of the information of all individual subsystems is thought to be a measure of order for the system. Correspondingly, equation (3) shows that the entropy is a measure of 
disorder for the system. If we regard the whole universe as an isolated system, the second law of thermodynamics seems to show that the universe shall become more and more disorderly. This is the so-called heat death of the universe, and is worried by many physicist. In the following, we will see this is a paradox only, and may be resolved. As shown in our proof of the second law of thermodynamics, when one observes the system and finds the entropy increase, he has redefined the statistic ensemble under research, and changed the information of the system from $\mathcal{I}$ to $\mathcal{I}_{0}$. The decrease of $\mathcal{I}_{0}$ compared with its value found in the last observation makes the entropy increase. However, the redefinition of the ensemble by the observer is subjective. For the objective evolution of the original ensemble, the information $\mathcal{I}$ is conserving. The entropy increase, it is the decrease of $\mathcal{I}_{0}$, is compensated by the increase of the correlation information

$$
\mathcal{I}_{c} \equiv \mathcal{I}-\mathcal{I}_{0}
$$

between individual subsystems of the system, although it is ignored in the presentation of the second law. For a given ensemble in its time evolution, the more suitable measure of the order should be the (total) information $\mathcal{I}$. It does not change in time. From this view we see, that an isolated system will not become more and more disorderly. The universe, even if it may be thought to be an isolated system, will not approach the absolute disorder. The paradox of heat death is therefore resolved.

The second law of thermodynamics may be derived mathematically from the information conservation, means it is absolutely true in the present physics. There are a lot of conservation laws in the present physics, examples are energy conservation, momentum conservation, angular momentum conservation, ....and so on, they are of firm foundation and related with various symmetries in time, space and matter. Information conservation is also related with the symmetry of the physics. It is the symmetry under the unitary transformation in quantum theory, or the symmetry under the canonical transformation in classical theory. However, these two symmetries are the most fundamental symmetries in the quantum and classical theories respectively. It means that one may image a fundamental quantum/classical theory without time translation symmetry and therefore is not energy conserving, but any fundamental quantum/classical theory must be symmetric under the unitary/canonical transformation and therefore is information conserving. In this sense, the principle of information conservation, and therefore the second law of thermodynamics, is the most fundamental law in physics. The foundation of the information conservation is even wider than the unitary/canonical symmetry. As we have shown before, if independent states of a system evolve continuously into independent states, the dynamics is information conserving. One may wonder what will be the future (post quantum and post relativistic) physics, which kind of law will still hold in it? From the above analysis we see, the most hopeful one is the information conservation, or the second law of thermodynamics.

The above argument also offers a new form of representation for physics, in which, among the fundamental principles, the second law of thermodynamics is substituted by the principle of information conservation. The second law itself becomes a deduced inequality. At least, from 
the view point of aesthetics, this form is attractive. It is more symmetric. All quantitative fundamental principles are presented in the form of equations. All inequalities are deduced. Moreover, this form may be easier to use in the research of ensemble evolution between two successive observations, especially on the transfer of information from $\mathcal{I}_{0}$ to $\mathcal{I}_{c}$. I am also happy to note, that this form has already been dreamed by some one else[5].

Our proof of entropy increase relies only on the information conservation, therefore is valid not only for physics, but also for any statistical process, in which the information conservation is true. In this kind of process, the entropy of an isolated system always increases until reaches the equilibrium. This is important since the entropy increase may point out the direction of development. To find out these kinds of processes in biology, engineering, social science, and financial science would therefore be interesting

\section{Discussions}

Some people may be surprised, since they feel the second law of thermodynamics should be approximate only. They could not believe an exact irreversible law may be founded on a reversible dynamics. There are indeed papers discuss its possible violation. One example is connected with the fluctuation theory $[6,7]$. This is an interesting and valuable theory, but I am afraid that authors' declaration on the violation of the second law of thermodynamics may be wrong. The appearance of the entropy consuming trajectories is declared to be the evidence of the second law violation. However, the second law requires that the entropy does not decrease only for isolated systems. But systems which move along the entropy consuming trajectories in their examples are not isolated, but interacting with the surrounding medium.

The only possible objection against the above argument may be that, the observation contains subjective element, and the content of physics should be purely objective. This is a classical point of view in philosophy. We would say yes, physicists make every effort try to find out the truth of the objective world, but the only way to do this is by their observation. To extract the objective truth from observation, one needs a theory of the observation, at least he has to know the role played by the observation in his research. One element in observation is the physical interaction between the observed system, the measuring instrument, and the observer. This is purely objective, and is governed by physical laws. Another element is the decision of the observer on the kind of observation to be performed, and the changing of his mind after the observation. This is subjective. The later includes the possible redefinition of the statistical ensemble for further research. These two elements are nature and necessary for an observation. The above argument shows the role played by the observation in understanding the statistical irreversibility. Behind it one sees the purely objective reversible dynamics.

Some people show the violation of the second law by use of effective theories, such as FokkerPlanck equation, and by use of various approximate methods. This is not reliable. Theoretically, to prove or disprove a law as fundamental as the second law of thermodynamics, one has to use 
the fundamental theory and rigorous mathematical method.

The early attempt of proving the second law of thermodynamics was made by Boltzmann in nineteenth century. In the course, he proposed and proved his famous H-theorem. In doing so, he used the fundamental classical equation of motion and rigorous mathematical method. At that time, his work was almost perfect, except that it was based on a model of colliding particle system for the macroscopic matter, which is not general enough even from the view point of classical physics. Now, more than a century passed, some improvement is needed. We should abandon any special model for the matter, in order to make the proof general. The quantum theory must be fully considered. It means we should put the proof entirely on the basis of fundamental principle only, which should be true both in classical and in quantum physics. This is what we have done in our proof.

This work is supported by the National Nature Science Foundation of China with Grant number 10875003.

\section{References}

[1] Q.-R. Zhang A general information theoretical proof for the second law of thermodynamics Int. J. Mod. Phys. E17 531 (2008)

[2] Q.-R. Zhang Axiomatic foundations for the principle of entropy increase Int. J. Mod. Phys. E17 1075 (2008)

[3] A. Feinstein Foundation of Information Theory (New York, London; McGraw-Hill 1958)

[4] L. D. Landau and E. M. Lifshitz Statistical Physics, 3rd edition (Butterworth Heinemann, Oxford, 1980)

[5] T. Stonier Information as a basic property of the universe Biosyst. 38135 (1996)

[6] G. M. Wang, E. M. Sevick, E. Mittag, D. J. Searles, and D.J. Evans Experimental demonstration of violations of the second law of thermodynamics for small systems and short time scales Phys. Rev. Lett. 89050601 (2002)

[7] D. J. Evans, E. G. D. Cohen, G. P. Morriss Probability of second law violations in shearing steady states Phys. Rev. Lett.71 2401 (1993) 